\title{
MULTIPLE ARX MODEL-BASED AIR-FUEL RATIO PREDICTIVE CONTROL FOR SI ENGINES
}

\author{
Tomáš Polóni *, Boris Rohal'-Ilkiv*, \\ Tor Arne Johansen** \\ * Department of Automation, Information and \\ Instrumentation Technology, Faculty of Mechanical \\ Engineering, Slovak University of Technology, Bratislava, \\ Slovakia \\ ** Department of Engineering Cybernetics, Norwegian \\ University of Science and Technology, Trondheim, Norway \\ fax : +421252495315 and e-mail : tomas.poloni@stuba.sk
}

\begin{abstract}
In this article the predictive control is suggested to control the injection fuel pulse width in such a manner that the air-fuel ratio deviates as little as possible from the stoichiometric ratio during the transients of the engine. The applied control strategy is based on the knowledge of an internal model of the airpath, predicting the change of the air flow through cylinders, and consequently, setting the prediction profile of the desired values of the objective function. The second modeled subsystem of the fuel-path is an explicit component of the objective function where the amount of the fuel is a function of the control action. It was demonstrated by simulation that multiple model predictive control has the potential to compete with standard look-up table strategies. Thus with further research in the predictive control of air-fuel ratio, cleaner exhausts may be expected.
\end{abstract}

Keywords: Air-fuel ratio, multiple model predictive control, nonlinear parameter varying system

\section{INTRODUCTION}

The problem of air-fuel ratio (AFR) control is one of the main parts of the more complex emission reduction strategy for combustion engines. The mixture quality is essential for efficiency of a threeway catalytic converter and therefore sufficient control techniques are needed to fulfil emission legislations. During the last twenty years different control methodologies were developed from simple to more sophisticated "model (observer)based" ones. In advanced control methods the model plays the most important role in the control strategy. A classical approach to modeling problem of AFR is based on linear observer theory where physical models of the process are part of state estimator (Guzzella and Onder, 2004) (Powell et al., 1998). A review of observers based on physical laws related to "gray-box" models can be found in (Hendricks and Luther, 2001). Another promising branch of control model-based strategies relies on "black-box" modeling principles where identified models are used. From the field of nonlinear approximation theory many dif- 
ferent nonlinear model structures have been applied to engine emission control problems. One of the most popular approaches to combustion engine modeling is based on neural network principles for their flexibility (Nelles, 2001). Especially, the AFR modeling problem was solved by radial basis function observer in (Manzie et al., 2002), by Chebyshev polynomial network in (Gorinevsky et al., 2003) and recently a simulator of AFR dynamics based on recurrent neural network was proposed by (Arsie et al., 2006). The purpose of this study is to design the AFR predictive controller based on linear parameter varying model (LPV) of the AFR and a simulative verification of its ability to maintain stoichiometric mixture during transients throughout different operating regimes of the 2.8 liter engine.

\section{MODEL STRUCTURE}

This section closely describes the model structure. First, a general weighted linear local model with single input single output (SISO) structure is presented. Specifically, composite local linear ARX models with weighted validity (Murray-Smith and Johansen, 1997) are identified to model AFR nonlinear dynamics. The global AFR model is then validated against measured data (Polóni et al., 2007). Weighted linear local models (LLM) have already been used in engine emission NOx control applications as an extension of radial basis function network sometime referred to as local linear neuro-fuzzy tree network (Isermann and Müller, 2003) (Hafner et al., 1999) and also in diesel engine drivetrain modeling (Johansen et al., 1998). Below it is shown how this structure can be applied for modeling of AFR dynamics of the engine.

\subsection{Weighted linear local model network structure}

The basic principle of this nonlinear modeling technique is in partitioning the operating regimes. For these operating regimes LLMs are defined. The transition between particular local models is fluent due to smooth interpolation (weighting) functions. In this case the local models will be linear ARX models with weighted parameters in an operating point $\phi \in \Phi \subset \mathbb{R}^{n_{\phi}}$,

$$
\begin{aligned}
& \sum_{h=1}^{n_{M}} \rho_{h}(\phi(k)) A_{h}(q) y(k)=\sum_{h=1}^{n_{M}} \rho_{h}(\phi(k)) B_{h}(q) u(k)+ \\
& +\sum_{h=1}^{n_{M}} \rho_{h}(\phi(k)) c_{h}+e(k)
\end{aligned}
$$

defining polynomials $A_{h}$ and $B_{h}$

$$
\begin{aligned}
& A_{h}(q)=1+a_{h, 1} q^{-1}+\ldots+a_{h, n_{y}} q^{-n y} \\
& B_{h}(q)=b_{h, 1+d_{h}} q^{-1-d_{h}}+\ldots+b_{h, n_{u}+d_{h}} q^{-n u-d_{h}}
\end{aligned}
$$

where $a_{h, i}, b_{h,\left(j+d_{h}\right)}, c_{h}$ are the h-th local function parameters and $d_{h}$ is the delay. The parameters $n_{M}$ and $n_{\gamma}$ stand for the number of local models and size of the regression vector (8) respectively. Here $q^{-1}$ is the time shift operator, i.e. $q^{-i} y(k)=y(k-i)$. The Gaussian local model validity function $\left\{\tilde{\rho_{h}}: \Phi \rightarrow(0,1)\right\}_{h=1}^{n_{M}}$ is defined by the vector of center $\mathbf{c}_{c, h} \in \mathbb{R}^{n_{\phi}}$ and by the scaling matrix $\mathbf{M}_{h}$

$$
\begin{aligned}
\tilde{\rho_{h}}(\phi(k)) & =e^{-\left(\phi(k)-\mathbf{c}_{c, h}\right)^{T} \mathbf{M}_{h}\left(\phi(k)-\mathbf{c}_{c, h}\right)} \\
\mathbf{M}_{h} & =\left(\begin{array}{cccc}
\frac{1}{\sigma_{h, 1}^{2}} & 0 & \cdots & 0 \\
0 & \frac{1}{\sigma_{h, 2}^{2}} & \cdots & 0 \\
\vdots & \vdots & \ddots & \vdots \\
0 & 0 & \cdots & \frac{1}{\sigma_{h, n_{\phi}}^{2}}
\end{array}\right)
\end{aligned}
$$

The function $\tilde{\rho_{h}}$ can be considered as degree of fulfilment (even though it is not a combination of antecedent fuzzy sets). To achieve a partition of unity, local model validity functions are normalised to get the weighting functions used which is based on Takagi-Sugeno fuzzy inference (Takagi and Sugeno, 1985).

$$
\rho_{h}(\phi(k))=\frac{\tilde{\rho_{h}}(\phi(k))}{\sum_{h=1}^{n_{M}} \tilde{\rho_{h}}(\phi(k))}
$$

That means in any operating point $\sum_{h=1}^{n_{M}} \rho_{h}(\phi(k))=$ 1. For simulation of the model (1) following equation has to be considered

$$
\begin{aligned}
y_{s}(k)= & \sum_{h=1}^{n_{M}} \rho_{h}(\phi(k))\left(\sum_{i=1}^{n y} \hat{a}_{h, i} q^{-i} y_{s}(k)+\right. \\
& \left.+\sum_{j=1}^{n u} \hat{b}_{h,\left(j+d_{h}\right)} q^{-j-d_{h}} u(k)+\hat{c}_{h}\right)
\end{aligned}
$$

Introducing the estimated parameter vector $\hat{\theta}_{h}$ and the regression vector $\gamma(k)$ with $d_{\max }=$ $\max \left\{d_{h}\right\}_{h=1}^{n_{M}}$

$$
\begin{aligned}
& \begin{aligned}
\hat{\theta}_{h}= & {\left[\hat{a}_{h, 1}, \hat{a}_{h, 2}, \ldots, \hat{a}_{h, n y},\{0,0, \ldots, 0\}_{d_{h}},\right.} \\
& \hat{b}_{h, 1+d_{h}}, \hat{b}_{h, 2+d_{h}}, \ldots, \hat{b}_{h, n u+d_{h}},
\end{aligned} \\
& \left.\{0,0, \ldots, 0\}_{d_{\max }-d_{h}}\right]^{T} \\
& \gamma(k)=\left[-y_{s}(k-1),-y_{s}(k-2), \ldots,-y_{s}(k-n y),\right. \\
& \left.u(k-1), u(k-2), \ldots, u\left(k-n u-d_{\max }\right)\right]^{T}
\end{aligned}
$$

equation (6) becomes,

$$
y_{s}(k)=\gamma^{T}(k) \sum_{h=1}^{n_{M}} \rho_{h}(\phi(k)) \hat{\theta}_{h}+\sum_{h=1}^{n_{M}} \rho_{h}(\phi(k)) \hat{c}_{h}
$$

The offset term $c_{h}$ of the local ARX model can be computed from the system's steady state values 


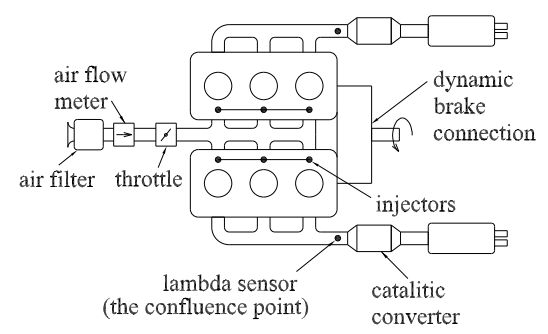

Fig. 1. Engine setup

$y_{e, h}, u_{e, h}$. Given a parameter estimate $\hat{\theta}_{h}$, the estimate of $c_{h}$ is defined as follows

$$
\hat{c}_{h}=y_{e, h}+y_{e, h} \sum_{i=1}^{n y} \hat{a}_{h, i}-u_{e, h} \sum_{j=1}^{n u} \hat{b}_{h, j}
$$

There are several possibilities how to estimate the parameters and weights of model (1). This is discussed in (Johansen and Foss, 1993) and (Takagi and Sugeno, 1985).

\subsection{Air-Fuel ratio model structure}

In this section we briefly introduce the $\mathrm{AF}$ ratio model structure, see (Polóni et al., 2007) for further details. The nonlinear (parameter varying) model is needed mainly due to nonlinear throttle characteristic (Heywood, 1988) and delay-varying AFR dynamics. The dynamic model of AFR is based on a definition of a mixture as a ratio of air and fuel quantities in time instance (k). Since $\lambda(k)$ is a non-dimensional ratio the air and fuel quantities can be expressed in any physical units, even relative ones. It is convenient to express these quantities in the meaning of relative mass densities ( $[\mathrm{g} /$ cylinder $])$ telling us how much mass of air (or fuel) is concentrated per volume of one cylinder. The relative mass density of the mixture consists of relative air density $m_{a}(k)$ and relative fuel density $m_{f}(k)$ that define the mixture quality in a time instance $(\mathrm{k})$. The effect of mixture formation is transformed from the discrete event process (one combustion cycle) to continuous changes of AFR information due to mixing dynamics in the exhaust manifold. To scale the AFR at one for stoichiometric mixture $\left(\lambda_{s t}=1\right)$, we divide the ratio by the value of theoretical stoichiometric coefficient for gasoline fuel $L_{t h} \approx 14.64$, so the ratio is defined

$$
\lambda(k)=\frac{1}{L_{t h}} \frac{m_{a}(k)}{m_{f}(k)}[-]
$$

The $m_{a}(k)$ and $m_{f}(k)$ information can be indirectly measured with a delay at the confluence point (Figure 1). To model $\lambda(k)$, two different subsystems with independent inputs are considered. The air-path subsystem $\left(m_{a}\right)$ with a throttle position $\left(t_{r}\right)$ input as a disturbance variable (DV) and the fuel-path subsystem $\left(m_{f}\right)$ with an injection pulse width $\left(u_{f}\right)$ input as a manipulated variable (MV). The other DV is the engine speed $\left(n_{e}\right)$ which is implicitly included in the model to define the operating point together with $t_{r}$. In accordance with the general model structure presented in Section 2.1 the key variables are defined in Table 1. In the operating point vector

Table 1. Symbol connection between general expression and the model

\begin{tabular}{llll}
\hline general & air-path & fuel-path & operating \\
symbol & model & model & point \\
\hline$y(k)$ & $m_{a}(k)$ & $m_{f}(k)$ & \\
$u(k)$ & $t_{r}(k)$ & $u_{f}(k)$ & \\
$\gamma(k)$ & $\gamma_{a}(k)$ & $\gamma_{f}(k)$ & \\
$\hat{\theta}_{h}$ & $\hat{\theta}_{a, h}$ & $\hat{\theta}_{f, h}$ & \\
$\rho_{h}(\phi(k))$ & $\rho_{a, h}(\phi(k))$ & $\rho_{f, h}(\phi(k))$ & \\
$\hat{c}_{h}$ & $\hat{c}_{a, h}$ & $\hat{c}_{f, h}$ & \\
$\phi(k)$ & & & {$\left[n_{e}(k), t_{r}(k-\delta)\right]^{T}$} \\
\hline
\end{tabular}

the parameter $\delta$ represents the throttle position delay. To simulate the AFR dynamics we combine (9) with (11)

$$
\lambda_{s}(k)=\frac{1}{L_{t h}}\left[\frac{\gamma_{a}^{T}(k) \sum_{h=1}^{n_{A}} \rho_{a, h}(\phi(k)) \hat{\theta}_{a, h}+\sum_{h=1}^{n_{A}} \rho_{a, h}(\phi(k)) \hat{c}_{a, h}}{\gamma_{f}^{T}(k) \sum_{h=1}^{n_{F}} \rho_{f, h}(\phi(k)) \hat{\theta}_{f, h}+\sum_{h=1}^{n_{F}} \rho_{f, h}(\phi(k)) \hat{c}_{f, h}}\right]
$$

The weighting functions considered for the global AFR model are shown at Figures 2 and 3.

\section{PREDICTIVE CONTROLLER DESIGN}

The applied control strategy is based on the knowledge of an internal model ${ }^{1}$ (IM) of airpath, predicting the change of air flow through cylinders, and consequently, setting the profile of desired values of the objective function on the control horizon. The second modelled subsystem of the fuel-path is an explicit component of the

\footnotetext{
1 Implying from IM strategy, we write $y_{s}$ in (8) and (18) as internally simulated outputs
}

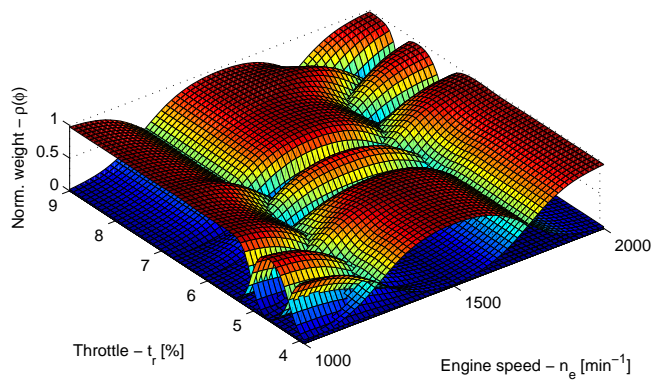

Fig. 2. Weighting function - air path 
objective function where the amount of the fuel is a function of optimized control action.

\subsection{Linear predictive model}

The predictive control can come out from several model structures of the system that lead to different computation algorithms of the control action (Maciejowski, 2002). Proposed MPC stands on linearised process model similarly used in (Roubos et al., 1999) and (Mollov et al., 2004). In this case we will consider the state space (SS) formulation of the system, therefore it is necessary to express linear local ARX models in parameter varying realigned SS model

$$
\begin{aligned}
x_{(a, f)}(k+1) & =A_{(a, f)}(\phi) x_{(a, f)}(k)+B_{(a, f)}(\phi) u_{(a, f)}(k) \\
m_{s,(a, f)}(k) & =C_{(a, f)} x_{(a, f)}(k)
\end{aligned}
$$

This is a non-minimal SS representation which advantage is, that no state observer is needed. The individual vectors and matrices of equation (13) are defined as follows

$$
A_{(a, f)}(\phi)=\left(\begin{array}{ccccccccccc}
-a_{1}(\phi) & -a_{2}(\phi) & \cdots & -a_{n y-1}(\phi) & -a_{n y}(\phi) & b_{2}(\phi) & \cdots & b_{n u-1+d_{\max }}(\phi) & b_{n u+d_{\max }}(\phi) & c(\phi) \\
1 & 0 & \cdots & 0 & 0 & 0 & \cdots & 0 & 0 \\
0 & 1 & \cdots & 0 & 0 & 0 & \cdots & 0 & 0 & 0 \\
\vdots & \vdots & \ddots & \vdots & \vdots & \vdots & \ddots & \vdots & \vdots & \vdots \\
0 & 0 & \cdots & 1 & 0 & 0 & \cdots & 0 & 0 & 0 \\
0 & 0 & \cdots & 0 & 0 & 0 & \cdots & 0 & 0 & 0 \\
0 & 0 & \cdots & 0 & 0 & 1 & \cdots & 0 & 0 & 0 \\
\vdots & \vdots & \ddots & \vdots & \vdots & \vdots & \ddots & \vdots & \vdots & \vdots \\
0 & 0 & \cdots & 0 & 0 & 0 & \cdots & 1 & 0 & 0 \\
0 & 0 & \cdots & 0 & 0 & 0 & \cdots & 0 & 0 & 1
\end{array}\right)(a, f)
$$

$x_{(a, f)}(k)=\left(y_{s}(k), y_{s}(k-1), \ldots, y_{s}(k-n y+1), u(k-1), u(k-2), \ldots, u\left(k-n u-d_{\max }\right), 1\right)_{(a, f)}^{T}$

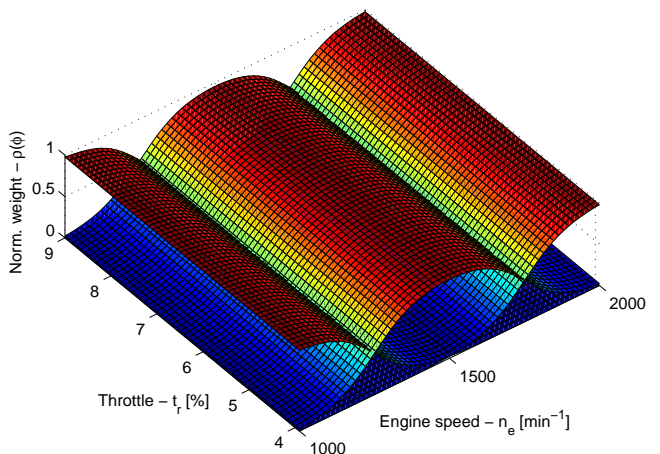

Fig. 3. Weighting function - fuel path

$$
\begin{gathered}
B_{(a, f)}(\phi)=\left(\begin{array}{llllllllllll}
b_{1}(\phi) & 0 & 0 & \ldots & 0 & 1 & 0 & \ldots & 0 & 0
\end{array}\right)_{(a, f)}^{T} \\
C_{(a, f)}=\left(\begin{array}{lllllllllll}
1 & 0 & \ldots & 0 & 0 & 0 & \ldots & 0 & 0 & 0
\end{array}\right)_{(a, f)}
\end{gathered}
$$

The parameters of multi-ARX models are scheduled by operating point $\phi(k)$ according to (9) and final weighted parameters are displayed in matrices $A_{(a, f)}$ and $B_{(a, f)}$ for both subsystems. The control of the fuel pulse width is tracking of the air mass changing profile on a prediction horizon from IM of the air-path, with the amount of injected fuel mass. Due to tracking offset elimination, the SS model of the fuel-path (13) (index $f$ ) is written in augmented SS model form to incorporate integral action

$$
\begin{gathered}
\tilde{x}_{f}(k+1)=\tilde{A}_{f}(\phi) \tilde{x}_{f}(k)+\tilde{B}_{f}(\phi) \Delta u_{f}(k)(18) \\
{\left[\begin{array}{c}
x_{f}(k+1) \\
u_{f}(k)
\end{array}\right]=\left[\begin{array}{cc}
A_{f}(\phi) & B_{f}(\phi) \\
0 & 1
\end{array}\right]\left[\begin{array}{c}
x_{f}(k) \\
u_{f}(k-1)
\end{array}\right]+} \\
+\left[\begin{array}{c}
B_{f}(\phi) \\
1
\end{array}\right] \Delta u_{f}(k) \\
m_{s, f}(k)=\tilde{C}_{f} \tilde{x}_{f}(k)+D_{f} \Delta u_{f}(k) \\
\text { or } \\
m_{s, f}(k)=\left[\begin{array}{ll}
C_{f} & D_{f}
\end{array}\right] \tilde{x}_{f}(k)+D_{f} \Delta u_{f}(k)
\end{gathered}
$$

Prediction of the air mass $\left(\underline{m}_{a}\right)$ on the prediction horizon $(N)$ is solely dependent on the throttle position $\left(\underline{t}_{r}\right)$ and is computed as

$$
\underline{m}_{\rightarrow}(k)=\Gamma_{a}(\phi) x_{a}(k)+\Omega_{a}(\phi) \stackrel{t}{\rightarrow}_{r}^{r}(k-1)
$$

Due to unprecise modeling (IM strategy), biased predictions of the air mass future trajectory and consequently fuel mass might occur. This error can be compensated by the term $L\left[\hat{m}_{f}(k)-\right.$ $\left.m_{s, f}(k)\right]$ in fuel mass prediction equation $\left(\underline{m}_{f}\right)$

$$
\begin{aligned}
\underline{m}_{f}(k) & =\Gamma_{f}(\phi) \tilde{x}_{f}(k)+\Omega_{f}(\phi) \Delta \underline{u}_{f}(k-1)+ \\
& +L\left[\hat{m}_{f}(k)-m_{s, f}(k)\right]
\end{aligned}
$$

The matrices of free response $\Gamma_{a}, \Gamma_{f}$ and forced response $\Omega_{a}, \Omega_{f}$ are computed from models (13) 
and (18) respectively (Maciejowski, 2002). Since there is only $\lambda(k)$ measurable in equation (11), the value of $m_{a}(k)$ needs to be substituted using IM of the air-path, then

$$
\hat{m}_{f}(k)=\frac{1}{L_{t h}} \frac{m_{s, a}(k)}{\lambda(k)}
$$

The estimate $\hat{m}_{f}(k)$ is used to compensate for possible bias errors of predicted $\underline{m}_{f}(k)$ in $(21)$.

\subsection{Computation of the control action}

The controller indirect setpoint is $\lambda=\lambda_{s t}=1$, and from (11) we define the control error

$$
m_{f}(k)-\frac{m_{a}(k)}{L_{t h}}=0
$$

The objective function for the AFR problem is then defined and written for chosen prediction horizon $N$ in matrix formulation

$J=\left[\underline{m}_{f}-\frac{m_{\rightarrow}}{L_{t h}}\right]^{T} Q\left[\underline{m}_{f}-\frac{m_{a}}{L_{t h}}\right]+\Delta \underline{u}_{f}^{T} R \Delta \underline{u}_{f}$

The control action computation stands on a minimization of the objective function

$$
\Delta \underline{u}_{f}=\arg \min _{\underline{u}_{u_{f}}} J
$$

For the sake of simplicity, the correction of the bias in (21) is omitted and analytical solution for constraint free case (Rossiter et al., 1998) is

$$
\begin{aligned}
\Delta \underline{u}_{f}= & -\left[\Omega_{f}^{T} Q \Omega_{f}+R\right]^{-1} . \\
& \cdot\left[\tilde{x}_{f}^{T} \Gamma_{f}^{T} Q \Omega_{f}-\left[\frac{\underline{m} a}{L_{t h}}\right]^{T} Q \Omega_{f}\right]^{T}
\end{aligned}
$$

Incremental controller can be expressed in the meaning of receding horizon as

$$
u_{f}(k)=u_{f}(k-1)+\Delta u_{f}(k)
$$

\section{SIMULATION}

The ability to control the mixture concentration at stoichiometric level is demonstrated through the simulation of an experimentally validated model (Polóni et al., 2007). The control scheme is shown in Figure 4. In the simulation the sudden changes of throttle position with changing load (see engine speed $\left(n_{e}\right)$ ) were considered to shift the operating regime. The nonlinear character mainly caused by the throttle can be seen at $m_{a}$, especially on different system gain in speed regimes around 1000 and $2000 \mathrm{~min}^{-1}$. Simulation results are displayed in Figure 5. The predicted $\lambda$ together with the prediction of $\frac{m_{a}}{L_{t h}}$ desired profile, tracked with predicted fuel mass $m_{f}$, are depicted in Figure 6 as well as the record of all computed predictions of the AFR during the simulation.

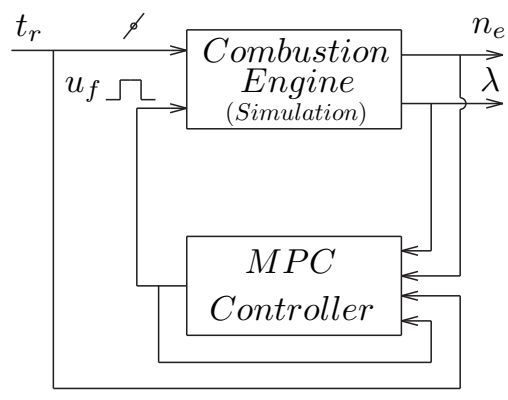

Fig. 4. Control scheme
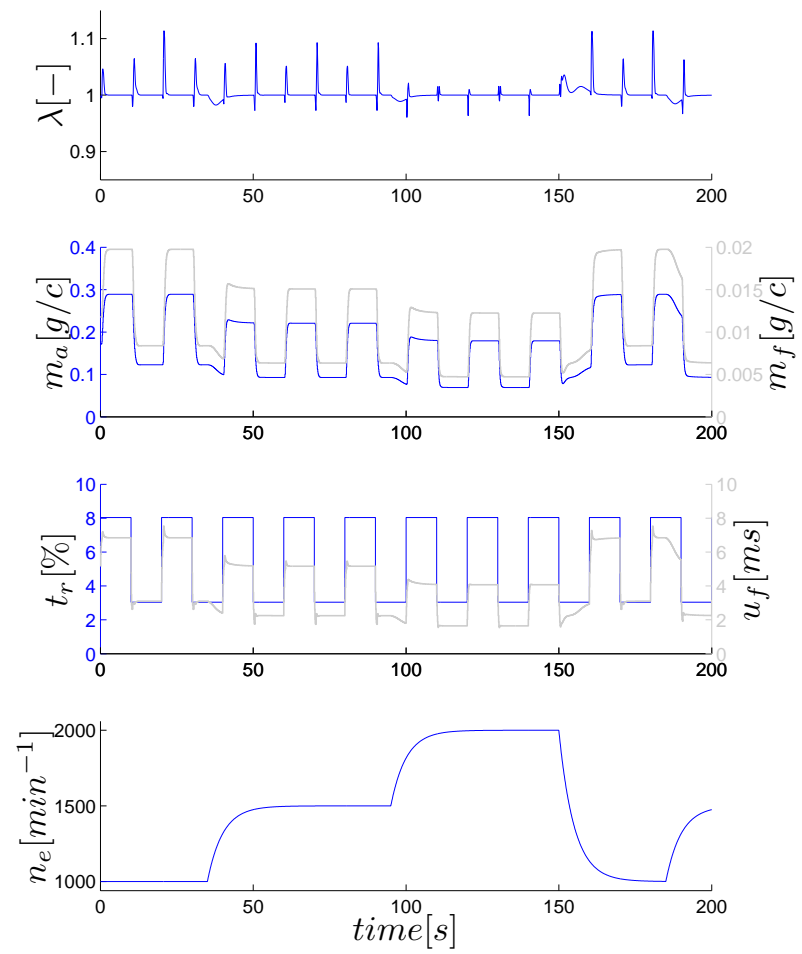

Fig. 5. Simulation of the engine air-fuel ratio control

\section{CONCLUSION}

In this article, we present preliminary design of a predictive controller for SI-engine air-fuel ratio. The control as well as the prediction are based on a ARX model network where the knowledge of physical phenomena is included a priori into assumptions that are utilized to design the model structure. The results are acceptable from the simulation point of view. However one has to expect worse results in real situation, particularly in $\lambda$ peek overshoots. The control is based on internal model (IM) simulation strategy, with throttle position measurement, without mass air flow sensor or intake manifold pressure sensor. For future real time applications the algorithm is expressed in a simple analytical form (without constraints) which brings rather lower computational demands on hardware. 

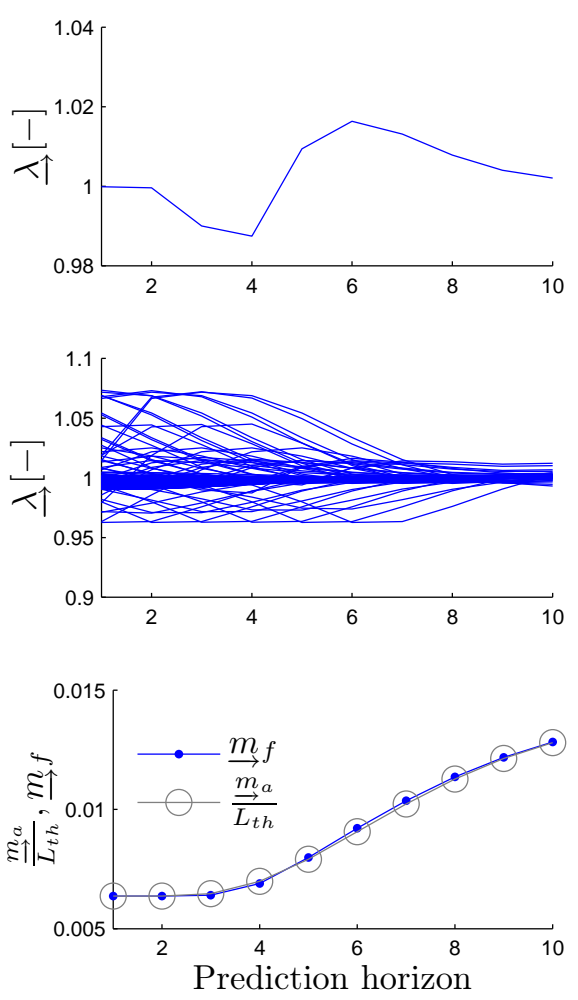

Fig. 6. Tracking fuel mass based on air mass setpoint on the prediction horizon

\section{ACKNOWLEDGMENTS}

The work has been supported by the grant agency APVV project number 0280-06. Part of the work was sponsored by the Research Council of Norway strategic university programme Computational Methods in Nonlinear Motion Control.

\section{REFERENCES}

Arsie, I., C. Pianese and M. Sorrentino (2006). A procedure to enhance identification of recurrent neural networks for simulating airfuel ratio dynamics in si engines. Engineering Applications of Artificial Intelligence 19, 6577.

Gorinevsky, D., J. Cook and G. Vukovich (2003). Nonlinear predictive control of transients in automotive vct engine using nonlinear parametric approximation. Transaction of the ASME (Journal of Dynamic Systems, Measurement, and Control) 125(3), 429-438.

Guzzella, L. and C. H. Onder (2004). Introduction to Modeling and Control of Internal Combustion Engine. Springer.

Hafner, M., M. Schuler and O. Nelles (1999). Dynamical identification and control of combustion engine exhaust. In: Proceedings of the American Control Conference. San Diego, California. pp. 222-226.
Hendricks, E. and J. B. Luther (2001). Model and observer based control of internal combustion engines. In: Proc. MECA (Modeling, Emissions and Control in Automotive Engines). Salerno, Italy.

Heywood, J. B. (1988). Internal Combustion Engine Fundamentals. McGraw-Hill.

Isermann, R. and N. Müller (2003). Design of computer controlled combustion engines. Mechatronics 13(10), 1067-1089.

Johansen, T. A. and B. A. Foss (1993). Constructing narmax models using armax models. International Journal of Control $\mathbf{5 8}(5), 1125-1153$.

Johansen, T. A., K. J. Hunt, P. J. Gawthrop and H. Fritz (1998). Off-equilibrium linearisation and design of gain-scheduled control with application to vehicle speed control. Control Engineering Practice 6(2), 167-180.

Maciejowski, J. M. (2002). Predictive Control with Constraints. Prentice Hall.

Manzie, Ch., M. Palaniswami, D. Ralph, H. Watson and X. Yi (2002). Model predictive control of a fuel injection system with a radial basis function network observer. Transaction of the ASME (Journal of Dynamic Systems, Measurement, and Control) 124, 648-658.

Mollov, S., R. Babuška, J. Abonyi and H. B. Verbruggen (2004). Effective optimization for fuzzy model predictive control. IEEE Trans. on Fyzzy Systems 12(5), 661-675.

Murray-Smith, R. and T. A. Johansen (1997). Multiple Model Approaches to Modelling and Control. Taylor\&Francis.

Nelles, O. (2001). Nonlinear System Identification. Springer.

Polóni, T., T. A. Johansen and B. Rohal'-Ilkiv (2007). Identification and modeling of air-fuel ratio dynamics of a gasoline combustion engine with weighted arx model network. Transaction of the ASME (Journal of Dynamic Systems, Measurement, and Control). Submitted.

Powell, J. D., N. P. Fekete and C. F. Chang (1998). Observer-based air-fuel ratio control. Control Systems Magazine,IEEE 18(5), 7283.

Rossiter, J. A., B. Kouvaritakis and M. J. Rice (1998). A numerically robust state-space approach to stable-predictive control strategies. Automatica 34(1), 65-73.

Roubos, J. A., S. Mollov, R. Babuška and H. B. Verbruggen (1999). Fuzzy model-based predictive control using takagi-sugeno models. International Journal of Approximate Reasoning 22(1), 3-30.

Takagi, T. and M. Sugeno (1985). Fuzzy identification of systems and its application to modeling and control. IEEE Trans. Systems, Man and Cybernetics 15, 116-132. 